\title{
Effect of infliximab on inflammatory bowel disease with Takayasu arteritis: case series and review of the literature
}

\section{$\operatorname{AUTHOR(S):~}$}

Minami, Naoki; Nakase, Hiroshi; Yoshino, Takuya; Yamada, Satoshi; Toyonaga, Takahiko; Honzawa, Yusuke; Matsuura, Minoru; Chiba, Tsutomu

\section{CITATION:}

Minami, Naoki ... [et al]. Effect of infliximab on inflammatory bowel disease with Takayasu arteritis: case series and review of the literature. Clinical Journal of Gastroenterology 2013, 6(3): 226-230

\section{ISSUE DATE:}

2013-06

URL:

http://hdl.handle.net/2433/190412

\section{RIGHT:}

The final publication is available at Springer via http://dx.doi.org/10.1007/s12328-0130387-9; This is not the published version. Please cite only the published version.; この論文 は出版社版でありません。引用の際には出版社版をご確認ご利用ください。 


\section{Effect of infliximab on inflammatory bowel disease with Takayasu arteritis}

\section{- Case series and review of the literature -}

Naoki Minami ${ }^{\text {a }}$, Hiroshi Nakase ${ }^{\mathrm{a}, *}$, Takuya Yoshino ${ }^{\mathrm{a}}$, Satoshi Yamada ${ }^{\mathrm{a}}$, Takahiko Toyonaga ${ }^{\mathrm{b}}$,

Yusuke Honzawa $^{\text {a }}$, Minoru Matsuura ${ }^{\text {a }}$, Tsutomu Chiba ${ }^{\text {a }}$

a. Department of Gastroenterology and Hepatology, Graduate School of Medicine, Kyoto University, Kyoto, Japan

b. The Third Department of Internal Medicine, Kansai Medical University, Hirakata, Japan

* Correspondence to Hiroshi Nakase, MD, PhD

Department of Gastroenterology and Hepatology, Graduate School of Medicine, Kyoto University, 54 Kawahara-cho, Shogoin, Sakyo-ku, Kyoto, 606-8507, Japan

Tel: +81-75-751-4319

Fax: +81-75-751-4303

E-mail: hiropy_n@kuhp.kyoto-u.ac.jp 


\section{Abstract}

Takayasu arteritis (TA) and inflammatory bowel disease (IBD) are chronic inflammatory disorders. The mechanisms underlying these diseases are not precisely known, but tumor necrosis factor alpha (TNF- $\alpha$ ) is considered to have an important role in the pathophysiology of both TA and IBD. Simultaneous occurrence of both TA and IBD is rare. Our first case was a 42-year-old woman with TA and inflammatory bowel disease unclassified. The patient was refractory to treatment with an immunomodulator, and infliximab (IFX) was started. After starting IFX, clinical remission was achieved and maintained for 2 years. The second case was a 34-year-old woman with TA accompanied by Crohn's disease. Because her abdominal symptoms relapsed despite treatment with an immunomodulator, IFX was started. Both diseases were well controlled for 2 years by scheduled maintenance therapy with IFX. Relapse of the TA required increased doses of IFX at shorter intervals, which relieved her symptoms.

From literature we identified and our cases, there were nine cases for which IFX was effective. They may demonstrate the efficacy of IFX for IBD with TA and emphasize the role of TNF- $\alpha$ in the pathophysiology of them. 


\section{Keywords}

Takayasu arteritis • Inflammatory bowel disease • Tumor necrosis factor alpha $\cdot$ Infliximab 


\section{Introduction}

Takayasu arteritis (TA) is a chronic inflammatory vasculitis of unknown etiology that affects the large vessels, predominantly the aorta and its main branches. Vessel inflammation leads to wall thickening, fibrosis, stenosis, and thrombosis formation. Various ischemic symptoms appear depending on the affected arteries, such as chest, limb, and abdominal pain [1, 2].

Although TA has been associated with other autoimmune diseases, such as glomerulonephritis, systemic lupus erythematosus, juvenile idiopathic arthritis, anterior uveitis, sarcoidosis, and Sweet's syndromes, the coexistence of TA and inflammatory bowel disease (IBD) is rare [1-3]. The pathogenesis of both TA and IBD remains unclear. A common pathomechanism between the two diseases, however, is conceivable, because treatment with anti-tumor necrosis factor alpha (TNF- $\alpha$ ) antibody often leads to a favorable clinical outcome of TA and IBD [4-7].

Several case reports have demonstrated the therapeutic effects of anti-TNF- $\alpha$ antibody in IBD with TA [6-10]. We herein report our two cases of IBD with TA successfully treated with infliximab (IFX) and discuss previously published reports of the efficacy and safety of IFX treatment for IBD with TA. 
Minami et al.

\section{Case Report}

\section{Case 1}

A 42-year-old woman suffering from TA with severe arterial stenosis was followed as an outpatient at the Department of Cardiovascular Medicine, Kyoto University Hospital. In April 2007, she complained of recurrent abdominal pain with hematochezia. Colonoscopic findings revealed multiple ulcerations from the ileocecal region to the ascending colon. Contrast-enhanced computed tomography revealed stenosis of the superior mesenteric artery root. We considered that her abdominal symptoms were due to ischemia of the superior mesenteric artery. Percutaneous transcatheter angioplasty of the superior mesenteric artery was performed but did not relieve her symptoms. Colonoscopic findings after percutaneous transcatheter angioplasty revealed no significant change. Although her colonoscopic findings mimicked ulcerative colitis (UC), the involved areas were inconsistent with UC (Fig. 1). Histological findings of biopsy specimens from colonic mucosa revealed chronic inflammatory features with no characteristic features of UC and Crohn's disease (CD). Fecal culture was negative for pathogenic microbes. Intranuclear inclusion body of cytomegalovirus was not detected in biopsy specimens. Contrast-enhanced computed tomography did not reveal any abnormality of her small intestine. Therefore, she was diagnosed with inflammatory bowel disease unclassified (IBDU) accompanied by TA. Administration of prednisolone (PSL) and mercaptopurine did not 
ameliorate her symptoms, and therefore IFX treatment was initiated. Following treatment with IFX, her abdominal symptoms subsided and clinical remission of her IBD was maintained by scheduled maintenance therapy with IFX. She was successfully tapered off the PSL and contrast-enhanced computed tomography revealed no new vascular lesions.

\section{Case 2}

A 34-year-old woman presented with anterior neck pain. Physical examination showed bruit of left carotid artery on auscultation. Contrast-enhanced computed tomography revealed stenoses of left carotid artery, aortic arch, celiac artery and superior mesenteric artery. These clinical and imaging findings met the criteria of TA described in the Japanese Circulation Society guideline. Therefore, she was diagnosed with TA and treated with oral administration of PSL. She complained of worsening diarrhea and body weight loss under the treatment with PSL $10 \mathrm{mg} / \mathrm{day}$. Colonoscopy revealed multiple ulcerations at the terminal ileum. Histologic findings of biopsy specimens obtained from the ulcerative lesions indicated noncaseating granuloma and led to a diagnosis of CD. She was started on $2250 \mathrm{mg} /$ day mesalazine and $50 \mathrm{mg} /$ day azathioprine (AZA) with continued PSL at $10 \mathrm{mg} /$ day. Although her abdominal symptoms subsided for 7 months after combined treatment with mesalazine and AZA, her symptoms recurred. Double-balloon enteroscopy revealed discontinuous longitudinal ulcers from the distal ileum to 
the ileocecal valve (Fig. 2). Because her CD disease activity was not controlled with conventional therapies, treatment with IFX was initiated. After starting $5 \mathrm{mg} / \mathrm{kg}$ of IFX, clinical remission of her CD was rapidly achieved and treatment with PSL could be tapered off completely. Although she exhibited no symptoms related to CD by a scheduled maintenance therapy with IFX for 2 years, laboratory data revealed gradually increasing serum C-reactive protein (CRP) levels. The uptake of fluorodeoxyglucose in the thoracic aorta wall in positron emission tomography suggested that the increased serum CRP levels were associated with TA activity (Fig. 3a). The dose of IFX was increased (from $5 \mathrm{mg} / \mathrm{kg}$ to $10 \mathrm{mg} / \mathrm{kg}$ ) and the dosing interval was shortened (from every 8 weeks to every 6 weeks) to normalize the CRP levels. The uptake of fluorodeoxyglucose in the thoracic aorta wall in positron emission tomography disappeared and double-balloon enteroscopy revealed the disappearance of multiple ulcerations in the distal ileum (Fig. 3b).

Characteristics and outcomes of the 9 patients with IBD and TA treated with anti-TNF- $\alpha$ agents

Including our 2 cases, there have been a total of 9 cases (8 women, 1 man) with IBD and TA successfully treated with anti-TNF- $\alpha$ agents (Table 1). There were seven cases with CD, one case with UC, and one case with IBDU. IBD preceded TA in 4 of the 9 cases and TA preceded IBD in 
our 2 cases. In the remaining 3 cases, IBD and TA were simultaneously diagnosed. Mean age at definitive diagnosis of both IBD and TA was 28 years (median 28 years, range 16-49 years). PSL was administered in all 9 cases and immunomodulators such as AZA, 6-mercaptopurine, and methotrexate were administered in 7 cases before treatment with IFX was initiated. In 8 of 9 cases, the clinical symptoms subsided following the administration of IFX and scheduled maintenance therapy with IFX. In the remaining 1 case, IFX was switched to adalimumab (ADA) because of an allergic reaction to IFX and the patient's clinical symptoms disappeared after switching to ADA. In 5 of 9 cases, PSL was completely tapered after treatment with the anti-TNF- $\alpha$ was initiated.

\section{Discussion}

Based on the clinical outcome of our 2 cases, IFX was effective for achieving clinical remission of IBD concomitant with TA. Additionally, IFX treatment inhibited the progression of TA. These findings indicate the possibility that TNF- $\alpha$ is mainly involved in the pathophysiology of IBD concomitant with TA.

The simultaneous occurrence of IBD and TA is considered rare [3, 8]. Reports on IBD concomitant with TA have increased recently, however, although the reason for this is unclear [3, 7-13]. Until now, there have been 56 IBD cases concomitant with TA including our two cases, 
and the majority of cases were treated with corticosteroid therapy [3, 6-13].

Treatment with corticosteroids is effective for patients with both IBD and TA, but long-term use of corticosteroids is not necessarily favorable because of the related side effects $[1,2,14]$. Therefore, treatment with immunomodulators is usually started to control inflammation from both diseases as well as other autoimmune diseases. Administration of anti-TNF- $\alpha$ antibody has been considered in recent cases that failed to respond to conventional treatment, including immunomodulators [5-7].

IFX treatment was administered in only a total of 9 of 56 cases, including our 2 cases [6-10]. Only one case required switching from IFX to ADA due to an allergic reaction related to IFX. In all cases, the symptoms of both IBD and TA were ameliorated by treatment with IFX or ADA. These data suggest that anti-TNF- $\alpha$ treatment for IBD concomitant with TA might lead to a clinically favorable outcome compared with other inflammatory diseases. In fact, in our case 2 presented here, exacerbated inflammation of TA during scheduled maintenance therapy with IFX was reduced by increasing the dose and administration interval of IFX. Notably, PSL could be completely tapered off in 5 of 9 cases after starting anti-TNF- $\alpha$ treatment.

HLA analysis revealed that HLA-B52 and DR2 are common genetic factors that predispose to TA and UC [13]. In contrast, there is no clear association between a common HLA genotype shared by both CD and TA, although both diseases are granulomatous in nature with an integral 
cell-mediated autoimmune component in the pathogenesis of these two diseases [3, 15]. Both IBD and TA share common features of autoimmune origin. In addition, TNF- $\alpha$ is thought to induce mucosal inflammation in IBD patients and the granulomatous process in the vessel walls of patients with TA. It is therefore likely that treatment with anti-TNF- $\alpha$ agents will be useful in abrogating the immunoinflammatory features of coexisting TA and IBD.

On the other hand, there are some reports of patients with IBD developing TA despite IFX treatment $[11,12]$. Because a subpopulation of patients with IBD also has TA subclinically, it is not surprising that TA would appear during treatment for IBD. The mechanism underlying the appearance of TA in IBD patients treated with anti-TNF- $\alpha$ agents, however, is unclear. In addition, because the present study is limited in that previous cases report a short-term follow-up, we cannot comment on the long-term efficacy and safety of IFX treatment, especially when it is necessary to increase the dose and administration interval.

In conclusion, this review of previous cases reports and our cases may demonstrate the efficacy of anti-TNF- $\alpha$ treatment for patients with coexisting IBD and TA, and provide support for treatment with anti-TNF- $\alpha$ agents in cases refractory to conventional therapies. Further follow-up studies with a greater number of patients are required to assess the long-term efficacy and safety of anti-TNF- $\alpha$ therapy. 
Minami et al.

\section{Acknowledgments}

None. 


\section{References}

1. Kerr GS, Hallahan CW, Giordano J, Leavitt RY, Fauci AS, Rottem M, et al. Takayasu arteritis.

Ann Intern Med 1994;120(11):919-29.

2. Johnston SL, Lock RJ, Gompels MM. Takayasu arteritis: a review. J Clin Pathol 2002; 55(7):481-6.

3. Kusunoki R, Ishihara S, Sato M, Sumita Y, Mishima Y, Okada M, et al. Rare case of Takayasu's arteritis associated with Crohn's disease. Intern Med 2011;50(15):1581-5.

4. Rutgeerts P, Sandborn WJ, Feagan BG, Reinisch W, Olson A, Johanns J, et al. Infliximab for induction and maintenance therapy for ulcerative colitis. $\mathrm{N}$ Engl $\mathrm{J}$ Med 2005;353(23):2462-76.

5. Hoffman GS, Merkel PA, Brasington RD, Lenschow DJ, Liang P. Anti-tumor necrosis factor therapy in patients with difficult to treat Takayasu arteritis. Arthritis Rheum 2004;50(7):2296-304.

6. Calderón R, Estrada S, Ramírez de la Piscina P, Salvador M, Zabaleta S, Enciso C, et al. Infliximab in a patient with refractory ileocolic Crohn's disease and Takayasu arteritis. Rev Esp Enferm Dig 2010;102(2):145-6.

7. Gecse K, Ruzsa Z, Nagy F, Wittmann T, Molnár T. Successful infliximab treatment in a patient with Takayasu arteritis associated with ulcerative colitis or migration does not 
override genetics. Inflamm Bowel Dis 2011;17(7):E69-70. doi: 10.1002/ibd.21731.

8. Kellermayer R, Jain AK, Ferry G, Deguzman MM, Guillerman RP. Clinical challenges and images in GI. Aortitis as a rare complication of Crohn's disease. Gastroenterology 2008;134(3):668, 898.

9. Judah JR, Hammond CJ, Polyak SF, Drane WE, Valentine JF. The coexistence of Crohn's disease and Takayasu arteritis: diagnosis and treatment of combined disease in three patients. Pract Gastroenterol 2009;3:50-8

10. Ratuapli S, Mazlumzadeh M, Gurudu S, Money S, Heigh R. Coexisting Crohn's disease and Takayasu's arteritis in two patients treated with anti-TNF- $\alpha$ therapies. Case Rep Gastroenterol 2010;4(1):35-40.

11. Katoh N, Kubota M, Shimojima Y, Ishii W, Matsuda M, Akamatsu T, et al. Takayasu's arteritis in a patient with Crohn's disease: an unexpected association during infliximab therapy. Intern Med 2010;49(2):179-82.

12. Horai Y, Satoru O, Lapalme-Remis S, Sumiyoshi R, Nakashima Y, Suzuki T, et al. Takayasu arteritis developing during treatment of ulcerative colitis with infliximab. Mod Rheumatol. 2012 Jun 21.

13. Morita Y, Yamamura M, Suwaki K, Mima A, Ishizu T, Hirohata M, et al. Takayasu's arteritis associated with ulcerative colitis; genetic factors in this association. Intern Med 
Minami et al.

1996;35(7):574-8.

14. Podolsky DK. Inflammatory bowel disease. N Engl J Med 2002;347(6):417-29.

15. Arnaud L, Haroche J, Mathian A, Gorochov G, Amoura Z. Pathogenesis of Takayasu’s arteritis: a 2011 update. Autoimmun Rev 2011;11(1):61-7 


\section{Minami et al.}

Figure legends

Figure 1

Colonoscopy image showing mimicked UC, including friability of mucosa with erythema and ulcerations from the terminal ileum to the ascending colon.

Figure 2

Double-balloon enteroscopy image showing the longitudinal ulcerations in the distal ileum.

Figure 3

a. Positron emission tomography image showing the uptake of fluorodeoxyglucose in the thoracic aorta wall. (arrow)

b. Positron emission tomography image showing the disappearance of fluorodeoxyglucose in the thoracic aorta wall. (left panel)

Double-balloon enteroscopy image showing the disappearance of multiple ulcerations in the distal ileum. (right panel) 


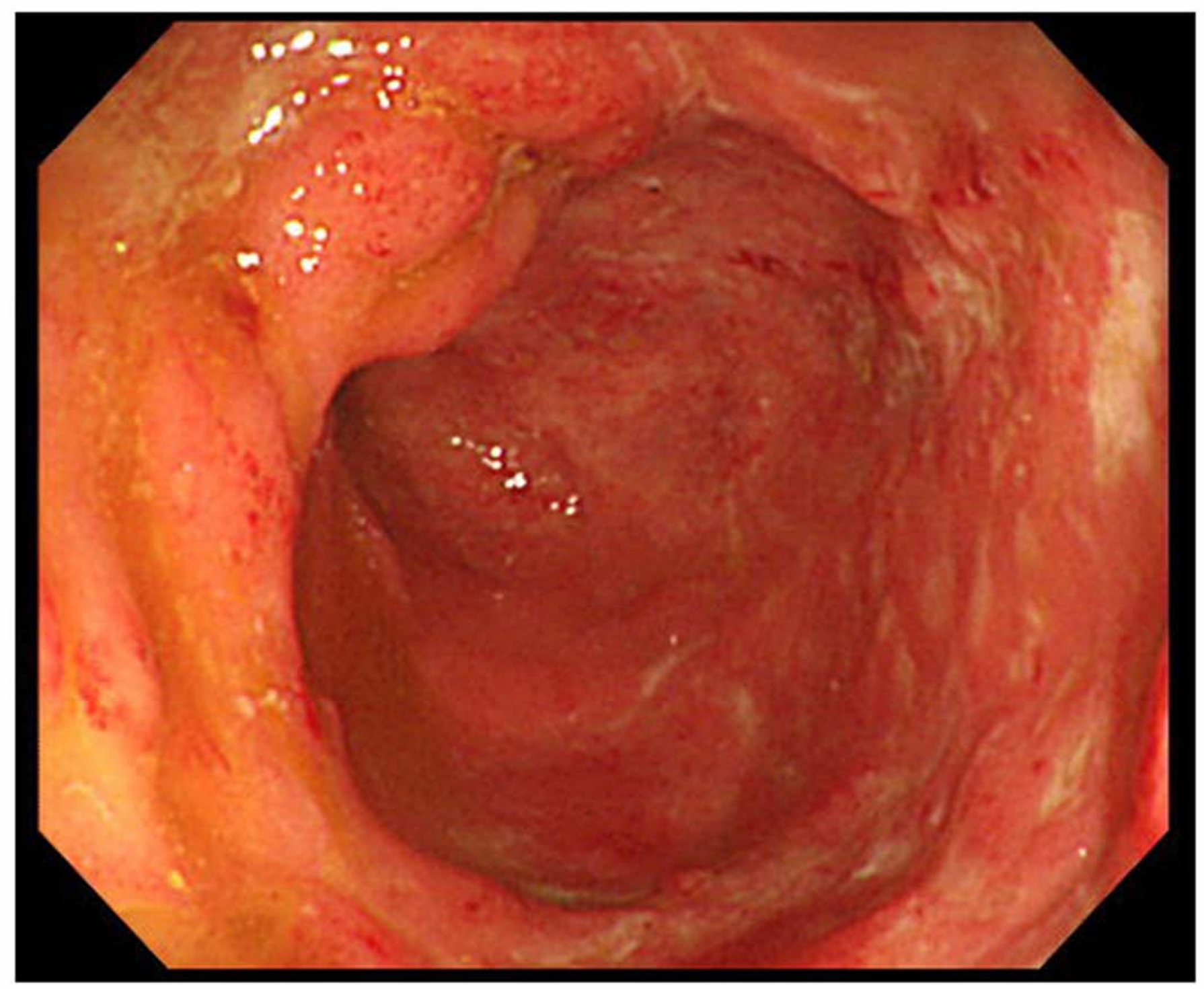

Figure 1 

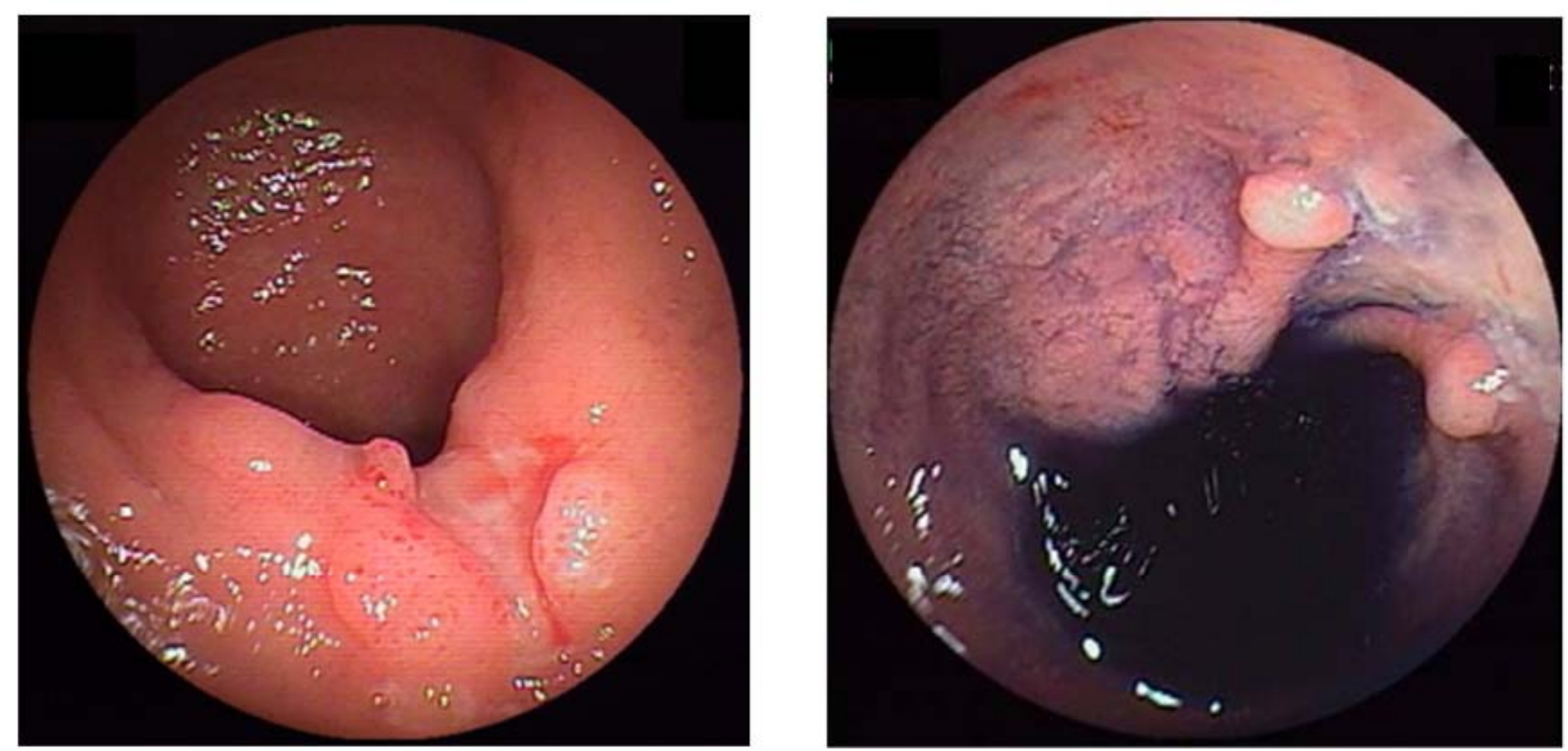

Figure 2 

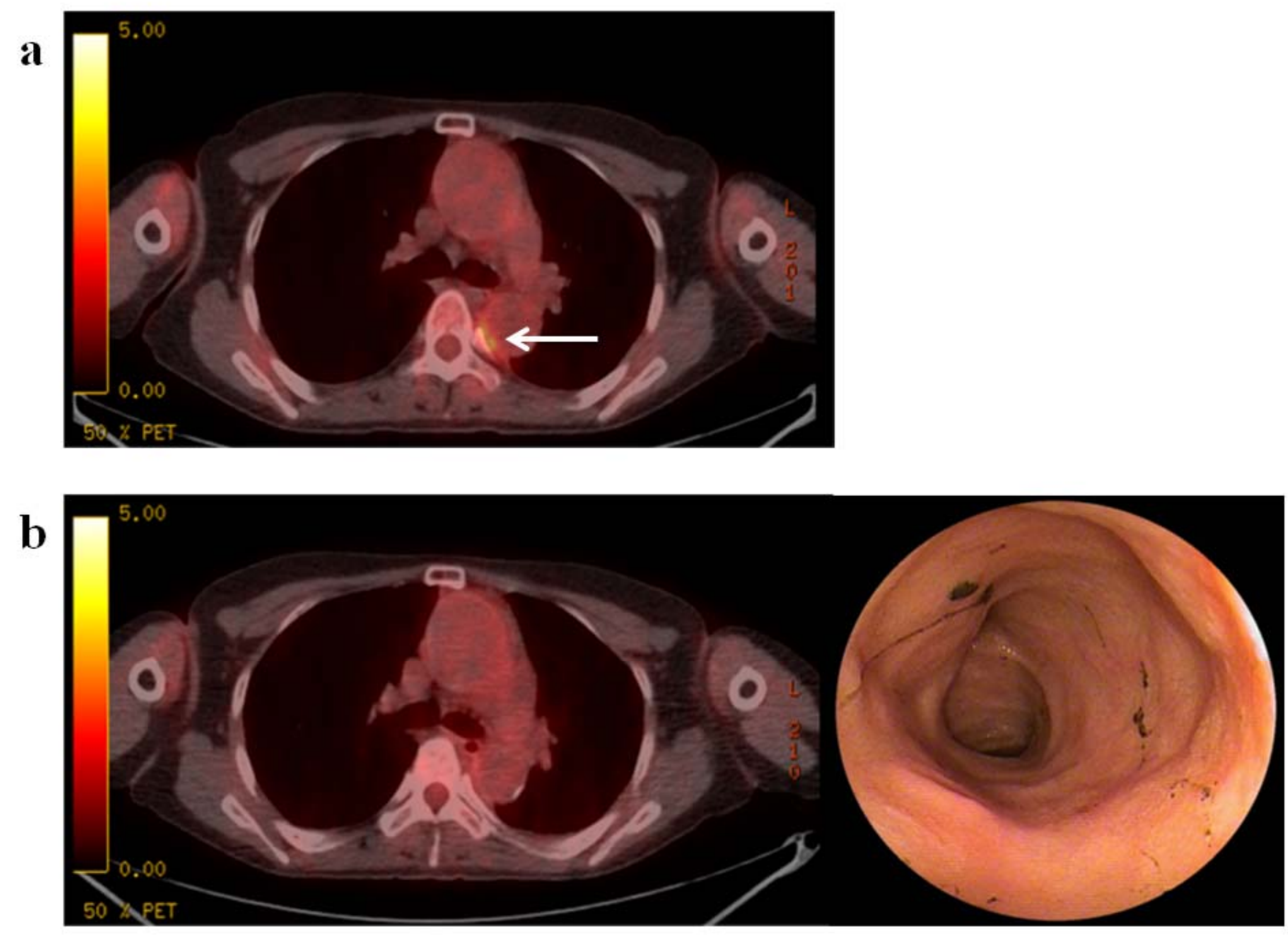

Figure 3 
Minami et al.

\section{Table}

Table 19 cases reports of IBD with TA treated successfully by IFX.

\begin{tabular}{|c|c|c|c|c|c|c|c|}
\hline \multirow{2}{*}{ Author } & \multirow{2}{*}{ Year } & \multirow{2}{*}{ Gender } & \multicolumn{2}{|c|}{ Age at diagnosis. } & \multirow{2}{*}{ Therapy } & \multirow{2}{*}{ PSL } & \multirow{2}{*}{ Reference } \\
\hline & & & IBD & TA & & & \\
\hline Kellermayer & 2008 & $\mathrm{~F}$ & $12(\mathrm{CD})$ & 17 & PSL, 5-ASA, 6-MP, IFX & discontinuation & 8 \\
\hline \multirow[t]{3}{*}{ Judah } & 2009 & $\mathrm{~F}$ & $24(\mathrm{CD})$ & 24 & PSL, IFX & discontinuation & 9 \\
\hline & & $\mathrm{F}$ & 30 (CD) & 49 & PSL, antibiotics, 6-MP, IFX & unknown & \\
\hline & & $\mathrm{F}$ & 30 (CD) & 30 & PSL, AZA, IFX, ADA & discontinuation & \\
\hline Calderon & 2010 & $\mathrm{~F}$ & 17 (CD) & 20 & PSL, 5-ASA, MTX, AZA, IFX & unknown & 6 \\
\hline Ratuapli & 2010 & M & $16(\mathrm{CD})$ & 16 & PSL, MTX, AZA, IFX & discontinuation & 10 \\
\hline Gecse & 2011 & $\mathrm{~F}$ & 20 (UC) & 30 & PSL, sulfasalazine, IFX & unknown & 7 \\
\hline \multirow[t]{2}{*}{ Our case } & 2012 & $\mathrm{~F}$ & 37 (IBDU) & 31 & PSL, 6-MP, IFX & discontinuation & \\
\hline & & $\mathrm{F}$ & $28(C D)$ & 22 & PSL, 5-ASA, AZA, IFX & continuation & \\
\hline
\end{tabular}

IBDU: inflammatory bowel disease unclassified, PSL: prednisolone, AZA: azathioprine,

6-MP: 6-mercaptopurine, MTX: methotrexate, IFX: infliximab,

ADA: adalimumab, 5-ASA: mesalazine 